\title{
Lipödem - Aktuelles zu einem weitgehend unbekannten Krankheitsbild
}

\author{
Lipoedema - New Facts of a Fairly Unknown Disease
}

Autor

Institut
W. Schmeller, I. Meier-Vollrath

Hanse-Klinik, Fachklinik für Liposuktion und operativ-ästhetische Dermatologie, Lübeck
Bibliografie

Dol $10.1055 / \mathrm{s}-2007-966651$

Akt Dermatol 2007; 33:

251-260 @ Georg Thieme

Verlag KG Stuttgart · New York ISSN 0340-2541

Korrespondenzadresse

Prof. Dr. med. Wilfried Schmeller

Hanse-Klinik

St.-Jürgen-Ring 66

23564 Lübeck

ws@hanse-klinik.com

\section{Zusammenfassung \\ $\nabla$}

Das Lipödem ist eine Erkrankung bei Frauen, charakterisiert durch eine umschriebene Unterhautfettvermehrung vorwiegend der Beine mit Ödemen, Spannungs- und Druckschmerzen sowie Hämatomneigung. Die Diagnose kann anhand klinischer Kriterien gestellt werden. Bisher war lediglich eine konservative Behandlung mittels

\section{Einleitung \\ $\nabla$}

Das Lipödem ist ein Krankheitsbild, welches immer noch vielen Laien - oft aber auch Ärzten und medizinischem Personal - nicht geläufig ist. Künstlerische Darstellungen, die heute als Lipödem interpretiert werden, gibt es bereits aus der Frühzeit der Menschheit. So existieren eine steinerne Statue der Großen Göttin im Tempel Hal Tarxien auf Malta von ca. 3000 v. Ch. ( $A$ abb. 1 a - im Vergleich mit einem klinischen Befund $\odot$ abb. 1 b) und ein Relief der Königin von Punt im ägyptischen Hatshepsut-Tempel in Deir el Bahari von ca. 1500 v. Chr. (๑ Abb. 1 c).

Die vorhandene Literatur zum Lipödem ist - im Gegensatz zum Lymphödem - insgesamt spärlich. Wissenschaftlich exakt beschrieben wurde die Krankheit erst 1940 von den amerikanischen Ärzten Allen und Hines [1]. Trotzdem wird die Entität dieses Krankheitsbildes in den USA vereinzelt immer noch angezweifelt; aus diesem Grund wurden sogar Publikationen zu diesem Thema abgelehnt (Schmeller, eigene Erfahrungen). Im neuen angloamerikanischen Schrifttum finden sich nur einzelne Fallberichte [2 -4]. Demgegenüber existiert im deutschen Sprachraum überwiegend im Bereich der Lymphologie - eine größere Zahl von Publikationen sowie eine Monographie [5]. In den Lehrbüchern der Dermatologie wird das Lipödem als Entität dargestellt [6-9]. In manchen Büchern wird es aber entwe- manueller Lymphdrainage, Kompression und Krankengymnastik möglich. Neuerdings kann operativ mittels Liposuktion in Tumeszenz-Lokalanästhesie (TLA) eine dauerhafte Verbesserung des Aussehens sowie eine Reduktion der Beschwerden mit einem deutlichen Gewinn an Lebensqualität erreicht werden. Die Kombination beider Verfahren gilt inzwischen als Therapiestandard.

der überhaupt nicht aufgeführt [10-12] oder falsch beschrieben [13,14]. Bei vielen Betroffenen wird daher erst nach einem jahrelangen Leidensweg die korrekte Diagnose gestellt und eine wirksame Therapie eingeleitet.

Seit einiger Zeit wird von Betroffenen bzw. von Selbsthilfegruppen im Internet relativ ausführlich über Symptome und Behandlungen informiert (www.lymphnetz.de, www.lipödem-liposuktion, www.Bundesverband-Lymphselbsthilfe. de). Nach Einführung neuer operative Therapiemöglichkeiten in den letzten Jahren wurde auch in wissenschaftlichen Zeitschriften wieder vermehrt über diese Krankheit berichtet [15].

\section{Definition \\ $\nabla$}

Das Lipödem ist charakterisiert durch eine chronisch progrediente, symmetrisch angeordnete Vermehrung des Unterhautfettgewebes mit orthostatischer Ödembildung. Betroffen sind überwiegend die unteren Extremitäten, teilweise in Kombination mit den Armen. Pathognomonisch sind Berührungs-, Druck- und Spannungsschmerzen sowie eine Neigung zu Hämatomen nach minimalen Traumen. Aus der Vielzahl der für das Lipödem benutzten Bezeichnungen sind die wichtigsten in $\bullet$ Tab. 1 aufgeführt. 


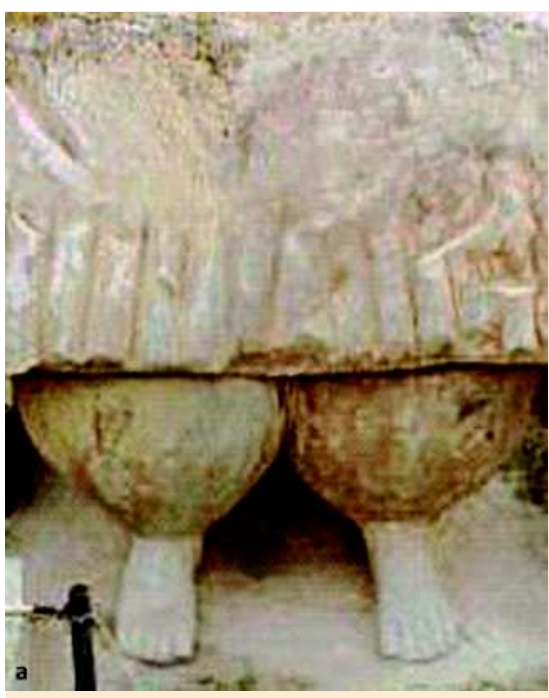

Abb. 1 Historische Darstellungen. a Ca. 5000 Jahre alte steinerne Statue der Großen Göttin im Tempel Hal Tarxien, Malta. b Klinisches Bild zum Vergleich. c Ca. 3500 Jahre altes Relief der Fürstin von Punt. HatschepsutTempel, Der el-Bahari, Ägypten (Umzeichnung nach Mariette).
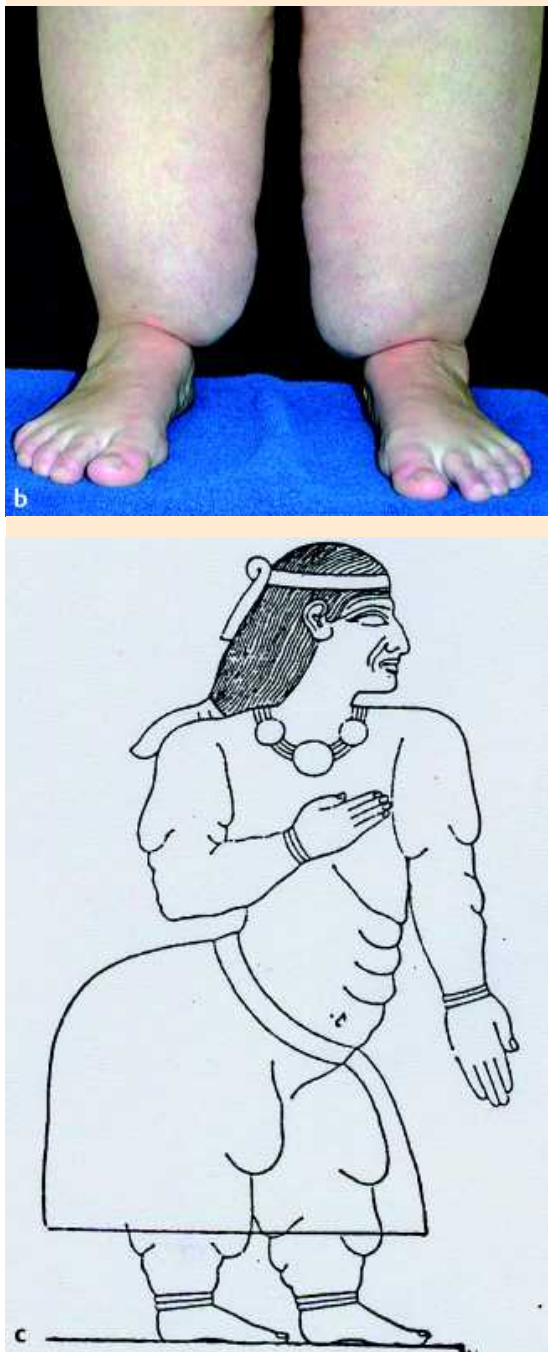

Tab. 1 Synonyme für das Lipödem
Lipalgie
Adiposalgie
Adipositas dolorosa
Lipomatosis dolorosa der Beine
Lipohypertrophia dolorosa
schmerzhaftes Säulenbein
schmerzhaftes Lipödemsyndrom

\section{Pathogenese \\ $\nabla$}

Die Ursache der Erkrankung ist unbekannt. Entscheidend sind sicher hormonelle Aspekte, da sich die Erkrankung fast ausschließlich bei Frauen findet. Männer sind nur in Ausnahmefällen, z.B. bei ausgeprägten hormonellen Störungen wie nach äthyltoxischer Leberzirrhose oder nach Hormontherapie, betroffen. Unseres Wissens gibt es lediglich einen Fallbericht über das Auftreten des Lipödems bei einem gesunden Mann [16].

Bezüglich des Krankheitsbeginns ergaben Untersuchungen an 119 Betroffenen aus den 1940er-Jahren eine weitgehend gleichmäßige Verteilung auf die einzelnen Lebensdekaden [17]. Demgegenüber wird in der neueren Literatur betont, dass die Erkrankung meist zur Zeit der Pubertät auftritt [18] oder sich nach Schwangerschaften manifestiert; bei 100 in den letzten Jahren ausgewerteten Patientinnen lag das Maximum des Auftretens des Lipödems im 3. und 4. Lebensjahrzehnt [19].

Neben hormonellen Einflüssen wird von einer genetischen Disposition ausgegangen, da häufig mehrere weibliche Mitglieder einer Familie betroffen sind. Die diesbezüglich aufgeführten Zahlen sind jedoch unterschiedlich und reichen von 16 bis 64 Prozent der Fälle $[1,17,20]$. Ob die vereinzelt beschriebenen nervalen Einflüsse in Form einer Schädigung des autonomen Nervensystems kausal von Bedeutung sind, ist unklar [21]. Dies gilt auch für Veränderungen der Mikrozirkulation [5, 21,22], von denen nicht sicher gesagt werden kann, ob es sich um ätiopathogenetisch relevante Veränderungen im Sinne einer so genannten Initialzündung oder um reine Sekundärphänomene handelt. Ebenfalls unklar ist, inwieweit beim Lipödem die bei Adipositas von Fettzellen freigesetzten Adipokine eine Rolle spielen [23]. Das Unterhautfettvolumen beim Lipödem ist - im Gegensatz zu dem beim Übergewicht - nicht „abhungerungsfähig“; auch treten beim Lipödem nicht die typischen Folgeschäden der Adipositas auf. Daher muss von anderen Rezeptoren und möglicherweise sogar von unterschiedlichen Fettzellen ausgegangen werden. Eine gleichzeitige Adipositas findet sich allerdings bei etwa 50 Prozent der Lipödempatientinnen [24].

\section{Pathophysiologie \\ $\nabla$}

Kausal liegen der Erkrankung mehrere Faktoren zugrunde. Optisch im Vordergrund steht - insbesondere bei schlanken Patienten - die Dysproportion zwischen Oberkörper und unterer Körperhälfte aufgrund der umschriebenen pathologischen Vermehrung des Unterhautfettgewebes. Ob es sich hierbei um eine Hypertrophie der Fettzellen, eine Hyperplasie oder eine Kombination von beidem handelt, ist immer noch unklar. Entscheidend ist ferner eine erhöhte Kapillarpermeabilität; sie führt zu einer vermehrten Flüssigkeits- und Eiweißansammlung im Interstitium und verursacht die orthostatischen Ödeme [22,25]. Deren Ausmaß - und nicht die absolute Fettmenge - ist für die Berührungs- und Druckempfindlichkeit des Gewebes entscheidend [18]. Eine verstärkte Kapillarfragilität bedingt die oft auffallende Hämatomneigung. Früher wurde vermutet, dass der zunehmende fett- bzw. ödembedingte Gewebedruck eine mechanische Abflussbehinderung von Lymphkapillaren, Präkollektoren und Kollektoren verursacht. Neuere Untersuchungen zeigten jedoch in frühen Stadien des Lipödems eine unbeeinträchtigte Funktion des Lymphsystems mit sogar erhöhtem Abtransport des vermehrten Flüssigkeitsvolumens. Bei den zunächst auftretenden passageren abendlichen Ödemen im Ober- bzw. Unterschenkel- 
bereich kann das intakte Lymphgefäßsystem das während des Tages vermehrt anfallende Volumen nicht mehr komplett abtransportieren (dynamische oder Hochvolumeninsuffizienz). Erst wenn die erhöhte lymphpflichtige Last jahre- bzw. jahrzehntelang die vorhandene Transportkapazität überschreitet, erfolgt eine Dekompensation [26,27] mit Erschöpfung der Funktionsreserven des Lymphgefäßsystems (mechanische oder Niedrigvolumeninsuffizienz). Hierzu tragen besonders die Sekundärveränderungen an den Lymphkollektoren bei. Als Folge der lang persistierenden Eiweißvermehrung im Interstitium entstehen abakterielle Entzündungen mit konsekutiver Verhärtung des Gewebes, wie dies auch beim Phlebödem in Form der Dermatoliposklerose bekannt ist. Die beim chronischen Lipödem sich entwickelnde perilymphovaskuläre Fibrose mit Lymphangiosklerose führt zu einer Verminderung des abtransportierten Volumens. In dieser späten Krankheitsphase besteht somit eine Kombination von erhöhtem Lymphanfall und vermindertem Abtransport. Dadurch entwickelt sich ein sekundäres Lymphödem in Form des Lipo-Lymphödems, was an einer Schwellung des Vorfußes erkennbar ist. Das Lipödem per se ist also primär keine lymphologische Erkrankung.

Es wird immer wieder betont, dass sich das Lipödem im Laufe von Jahrzehnten aus einer Lipohypertrophie (siehe unter Differenzialdiagnosen) entwickelt [28]; dem schmerzhaften Krankheitsbild des Lipödems würde somit ein schmerzloses Stadium der Lipohypertrophie vorangehen. Einerseits wird jedoch immer wieder beobachtet, dass sich auch Lipödeme schon ohne offensichtliche Vorstadien sehr früh in der Pubertät entwickeln können; andererseits bestehen die meisten Lipohypertrophien bis zum Lebensende ohne wesentliche Ödeme oder Schmerzen. Ob und wodurch das eine in das andere übergeht, ist unklar. Offensichtlich müssen relevante Zusatzfaktoren wie Genetik, Hormone - möglicherweise auch noch Zirkulationsstörungen und nervale Veränderungen - sowie das Lebensalter hinzukommen, um die krankheitsentscheidende Erhöhung der Kapillarpermeabilität und -fragilität und damit die charakteristische Symptomatik zu bewirken.

\section{Pathologische Anatomie \\ $\nabla$}

Die beim Lipödem histologisch nachweisbaren Veränderungen sind nicht pathognomonisch. Neben vermehrten und teils wohl auch hypertrophen Fettzellen zeigt sich im Interstitium ein hoher Gehalt kapillärer Blutgefäße; perivaskulär finden sich Makrophagen, Fibroblasten, Mastzellen sowie einzelne Fettgewebsnekrosen. In den Spätphasen der Erkrankung nimmt der fibrotische Anteil zu (22). Die histologischen Befunde sind dann weitgehend identisch mit denen der Dermatoliposklerose. Es handelt sich somit um das Endprodukt einer unspezifischen entzündlichen Gewebsreaktion, wie sie in vergleichbarer Weise z.B. bei der chronischen Veneninsuffizienz (CVI) vorkommt.

\section{Epidemiologie \\ $\nabla$}

Bezüglich der Häufigkeit existieren bislang keine exakten epidemiologischen Daten. In der Literatur wird ein Befall von $11 \%$ der weiblichen Bevölkerung in Deutschland vermutet [21]. Beim ausgesuchten Kollektiv einer lymphologischen Fachklinik in den Jahren 1995/96 fand sich bei etwa 15\% der stationären Patienten ein Lipödem (29). Eigene Nachfragen in vier deutschen
Lymphkliniken im Jahre 2003 ergaben einen vergleichbaren Anteil der Lipödeme in der Größenordnung von 8 bis 17\% [15].

\section{Diagnostik \\ $\nabla$}

Das Lipödem kann normalerweise anhand klinischer Kriterien eindeutig diagnostiziert werden. Typisch sind Zeitpunkt des Auftretens, symmetrische Verteilung der Fettpolster, Ödeme, Schmerzhaftigkeit bei Berührung und Druck sowie Hämatomneigung. Eine apparative bzw. invasive Diagnostik ist in der Regel nicht nötig. Besteht ein Lipo-Lymphödem, kann mittels bildgebender Verfahren (indirekte Lymphangiographie, FunktionsLymphszintigraphie) eine zusätzliche Quantifizierung morphologischer bzw. funktioneller Veränderungen des Lymphsystems erfolgen.

\section{Klinische Untersuchungsmethoden \\ $\nabla$}

Anamnese: Der Erkrankungsbeginn ist meist in der Pubertät, gelegentlich aber nach einer Schwangerschaft oder erst nach der Menopause. Manchmal wird über eine gleichzeitig einsetzende starke Gewichtszunahme berichtet; beim Stadium I des Lipödems liegt jedoch oft kein Übergewicht vor. Die betroffenen Frauen klagen über ein spontan auftretendes Spannungs- bzw. Schwellungsgefühl sowie über eine oft auffallende Berührungsund Druckschmerzhaftigkeit an Ober- und Unterschenkeln. Die Beschwerden verstärken sich meist im Laufe des Tages, insbesondere nach langem Stehen oder Sitzen. Ihre Ausprägung ist unabhängig von der Größe der Fettvermehrung. Zusätzlich werden meist abendliche Ödeme, vermehrt in der warmen Jahreszeit, angegeben. Berichtet wird ferner über bereits nach leichtem Anstoßen auftretende Einblutungen; Minitraumen, die bei Gesunden keinerlei Hautveränderungen bewirken, können an den lipödematösen Extremitäten Hämatome verursachen. Die Symptome sind an den Armen normalerweise geringer ausgeprägt als an den Beinen.

Die Betroffenen leiden ferner massiv unter Ihrem Aussehen; auf diese Problematik wurde bereits in den ersten Publikationen hingewiesen [1,17]. Viele Frauen berichten aufgrund des im Laufe der Zeit - trotz Sport und Diäten - zunehmenden Befundes über Frustrationen; diese führen dann häufig zu übermäßigem Essen mit folgender Gewichtszunahme. So ist die oft zusätzlich bestehende Adipositas nachvollziehbar. Sekundäre Lymphabflussstörungen in Form des Lipo-Lymphödems sind bei Übergewichtigen eher und häufiger zu finden als bei Normalgewichtigen.

Inspektion: Die meisten Patientinnen weisen eine deutliche Diskrepanz zwischen schlankerem Oberkörper und kräftiger unterer Körperhälfte auf. Bei ca. 97\% der betroffenen Frauen sind die Beine - Oberschenkel mit Hüften, oft zusätzlich noch die Unterschenkel - betroffen; bei ca. 30\% der Fälle findet sich ein Befund an den Oberarmen, evtl. auch an den Unterarmen bis zum Handgelenk [29].

Die Erkrankung ist chronisch und meist progredient. Die - Abb.2a-e zeigen unterschiedliche Schweregrade ( $\bullet$ Tab.2) und Lokalisationen ( $\odot$ Tab.3au.b). Anfangs findet sich eine gleichmäßig kleinknotig verdickte Subkutanschicht, wobei die Hautoberfläche makroskopisch glatt erscheint (Stadium I). Wenn die Knoten im Laufe der Zeit an Größe zunehmen, entstehen Unebenheiten der Hautoberfläche, die wellig wird oder 

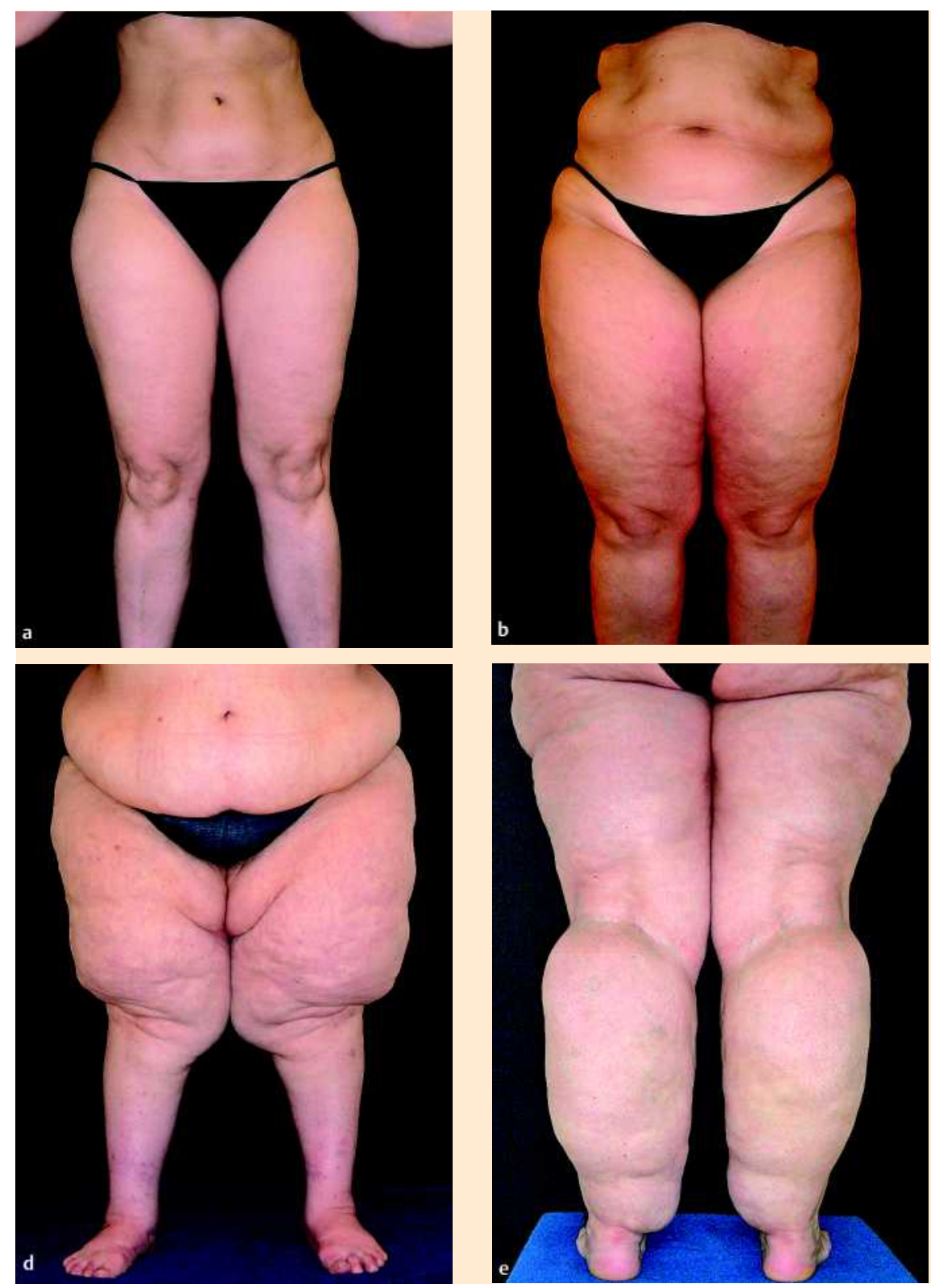

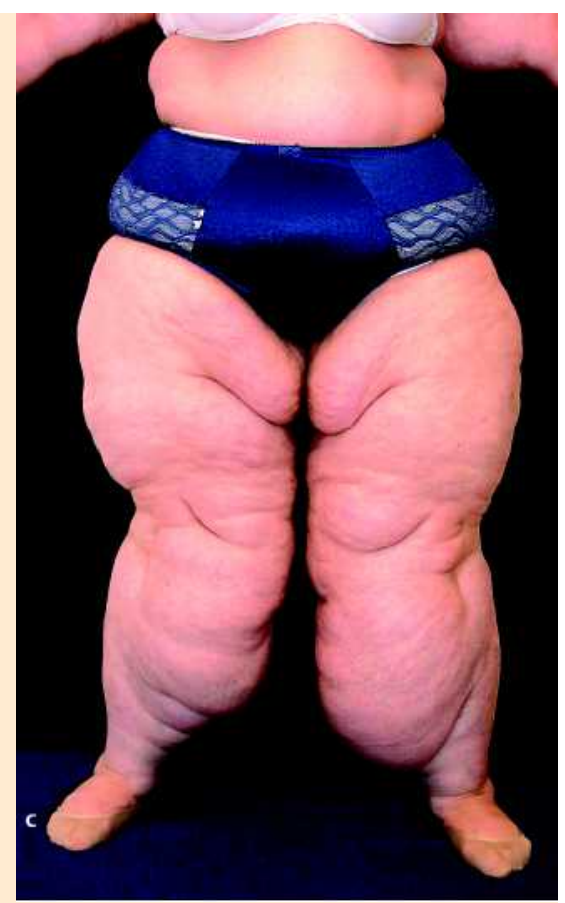

Abb. 2 Klinische Bilder. a Lipödem Stadium I (Ganzbeintyp). b Lipödem Stadium II (Ganzbeintyp). c Lipödem Stadium III (Ganzbeintyp). d Lipödem Stadium III (Oberschenkeltyp). e Lipödem Stadium III (Unterschenkeltyp). einer Orangenschale ähneln kann (Stadium II). Dies wird in Laienkreisen oft als „Cellulite“ bezeichnet. Bei fortgeschrittenen Formen wird das Subkutangewebe zunehmend indurierter; schließlich treten Lipom-ähnliche Knoten sowie lappige Fettwülste im Knie- und Oberschenkelbereich auf, die zu einer Behinderung beim Gehen führen können (Stadium III). Das betroffene Gewebe ist - meist in Abhängigkeit vom Ausmaß des Ödems - druckempfindlich. Eine Einteilung des Lipödems nach dem Ausmaß der Beschwerden oder Schmerzen existiert nicht. Funktionelle Veränderungen der Mikrozirkulation zeigen sich klinisch u. a. darin, dass die Haut an umschriebenen Stellen kühl ist. Oft bestehen Hämatome, ohne dass Traumen erinnerlich sind. In intertriginösen Bereichen subinguinal oder zwischen den Oberschenkeln können nässende Erosionen vorhanden sein. Beim Lipödem findet man keine Ödeme an Fußrücken oder Zehen. Wenn diese in späteren Verlaufsstadien als Folge einer sekundären Lymphabflussstörung auftreten (Lipo-Lymphödem), sind die Hautfalten an den Zehen verdickt und nicht abhebbar. Dieses so genannte Stemmersche Zeichen ist pathognomonisch für eine Beteiligung des Lymphsystems.
Palpation: Die umschriebene Fettvermehrung allein hinterlässt auf Druck keine Eindellungen; diese findet man nur bei einem deutlichen Begleitödem. Im Stadium I hat das Unterhautgewebe eine weiche Konsistenz und eine gleichmäßige oder auch feinknotige Struktur. Im Stadium II tastet man subkutan grobknotige Veränderungen. Im Stadium III ist der Befund noch ausgedehnter. An den Extremitäten kann die Volumenvermehrung abrupt oberhalb von Knöchel oder Handgelenk in einem Fettkragen enden; an den Beinen hat man dies früher als „Suaven-“ oder „Türkenhosenphänomen“ bezeichnet. Dabei finden sich umschriebene Fettvermehrungen, welche gelegentlich die Kniegelenkregionen schürzenförmig überlappen können. Meist bestehen deutliche Verhärtungen des Unterhautgewebes, besonders beim LipoLymphödem; als Ursache wird eine Fibrosklerose der interlobulären Septen angesehen [22]. 


\section{Apparative bzw. bildgebende Untersuchungs- methoden \\ $\nabla$}

Beim Lipödem können Lokalisation und Ausmaß der Fettvermehrung mittels Computertomographie (CT) und Magnetresonanztomographie (MRT) erfasst werden [30,31]. Auch mithilfe der Sonographie lassen sich quantitative und qualitative Aspekte des Fettgewebes erfassen. Während sich die normale Subkutis eher echoarm darstellt, ist sie beim Lipödem homogen verbreitert und weist zunächst eine gleichmäßig vermehrte Echogenität („Schneegestöber“) mit echoreichen Bindegewebssepten auf [32]. In späteren Stadien erscheinen zusätzlich echoarme Areale, wahrscheinlich aufgrund interstitieller Flüssigkeitsanreicherung. Bei der Kompressions-Sonographie ist der erzeugte Druckschmerz umso stärker und das Unterhautfettgewebe umso schwerer komprimierbar, je ausgeprägter das Ödem ist.

In der indirekten Lymphangiographie finden sich beim Lipödem geschlängelt verlaufende Lymphkollektoren und gefiederte bzw. flammenartige Kontrastmitteldepots, wie sie in ähnlicher Form aber auch bei Lymphödem und bei der Adipositas auftreten können [25]. Demgegenüber scheinen die mittels Fluoreszenz-Mikrolymphographie an betroffenen Extremitäten nachweisbaren sackförmigen oder fusiformen Mikroaneurysmen der Lymphkapillaren pathognomonisch zu sein [33]; ob ihnen jedoch eine Bedeutung im Rahmen der Pathogenese zukommt, ist unklar. Beim Lipo-Lymphödem können zusätzlich zu den oben aufgeführten Befunden - abhängig vom Ausmaß der Störungen am Lymphsystem - erweiterte initiale Lymphgefäße sowie geschlängelte Lymphkollektoren nachweisbar sein.

Zur Bestimmung einer evtl. vorhandenen Lymphabflussstörung kann die Funktions-Lymphszintigraphie eingesetzt werden. Dabei findet man beim reinen Lipödem oft keine oder nur geringe Veränderungen des epifaszialen Lymphtransports [34]. Der bereits beschriebene hyperdyname Lymphtransport in den frühen Stadien zeigt sich in Form von erhöhten Lymphknoten-UptakeWerten [20,27]. Bei der nach Jahren auftretenden Überlastung des Lymphgefäßsystems mit Ausbildung eines Lipo-Lymphödems lässt sich das Ausmaß der Lymphschädigung durch verlängerte Transportzeiten und einen erniedrigten Uptake in den regionalen Lymphknoten erfassen. Bedeutsam kann der Einsatz dieser Methode u. a. im Stadium I sein, um eine evtl. begleitende - und klinisch noch nicht sichtbare - Schädigung des Lymphgefäßsystems frühzeitig zu erkennen.

\section{Differenzialdiagnosen \\ $\nabla$}

Die wichtigste Differenzialdiagnose des Lipödems ist die Lipohypertrophie. Hierbei besteht - ebenfalls bei Frauen - anlagemäßig bedingt eine dysproportionierte Körperform aufgrund einer symmetrischen Unterhautfettvermehrung im Hüft- bzw. Beinbereich bei schlankem Rumpf. Die Ausdrücke Reit(er)hosen bzw. Reithosenadipositas bezeichnen die häufigste Formvariante; ein Befall der Arme ist selten. Während die morphologischen Veränderungen somit völlig gleich sein können, finden sich im Gegensatz zum Lipödem aber keine Ödeme. Daher weisen die Betroffenen auch keine Druck- oder Spannungsschmerzen auf. Somit besteht also auch keine behandlungsbedürftige Erkrankung, sondern allenfalls ein kosmetisches Problem. Es scheint aber Fälle zu geben, bei denen sich im Laufe der Zeit aus einer Lipohypertrophie ein Lipödem entwickelt [28].
Auch beim primären Lymphödem tritt beim weiblichen Geschlecht - überwiegend in der Pubertät - eine ein- oder beidseitige Schwellung der Beine auf; diese ist bei beidseitigem Befall immer asymmetrisch. Während die Schwellung beim Lymphödem jedoch typischerweise an den Zehen beginnt und erst später den Oberschenkel erreicht, tritt beim Lipödem die Umfangsvermehrung meist zuerst - oder gleichzeitig - am Oberschenkel auf. Beim Lymphödem ist aufgrund des Fußbefalls das Stemmersche Zeichen - im Gegensatz zum Lipödem - positiv. Druckschmerzhaftigkeit, Spannungs- bzw. Schweregefühl des Gewebes oder Hämatomneigung bestehen nicht. Kombinationen mit einem Lipödem (Lympho-Lipödem) können allerdings vorkommen.

Das Phlebödem ist ein Symptom der chronischen Veneninsuffizienz (CVI). Es kann bei beiden Geschlechtern ein- oder beidseitig auftreten und geht typischerweise den kutanen und subkutanen Veränderungen (Stauungsekzem, Hyperpigmentierung, Hypodermitis, Dermatoliposklerose, Ulzeration) voraus. Funktionsuntersuchungen wie Ultraschall-Doppler, Licht-ReflexionsRheographie und Duplex-Sonographie weisen pathologische Befunde auf. Allerdings existieren auch hier Mischformen (PhleboLipödem).

Beim Morbus Dercum (Lipomatosis dolorosa) haben die Betroffenen - meist übergewichtige Frauen mittleren Alters - ausgesprochen schmerzhafte, symmetrische Fettansammlungen an Rumpf oder Armen, aber vor allem an den Beinen; die Füße sind ausgespart. Im Gegensatz zum Lipödem manifestiert sich die Erkrankung aber häufig erst mit dem Beginn der Menopause. Es ist kein Ödem nachweisbar, manchmal wird eine Muskelschwäche angegeben. Häufig bestehen zusätzlich Alkoholabusus sowie psychische Veränderungen wie Reizbarkeit und Depressionen. Therapeutisch wurde bezüglich der Schmerzsymptomatik über gute Erfahrungen mit Lidocaininfusionen berichtet [35]. Unklar ist, ob der Morbus Dercum tatsächlich eine eigene Entität oder vielleicht nur eine Sonderform eines spät aufgetretenen Lipödems ist.

Bei der benignen symmetrischen Lipomatose Launois-Bensaude (Madelung-Krankheit) finden sich diffuse, teigig-derbe Unterhautfettvermehrungen. Bezüglich der Lokalisation unterscheidet man den Hals-Nacken-Typ (Typ I; Madelung-Fetthals, „Büffelhöcker“, lokalisierter Typ), den Schultergürteltyp (Typ II; pseudoathletischer Typ), den Beckentyp (Typ III; gynäkoider Typ) und den abdominellen Typ (Typ IV). Die Erkrankung betrifft Männer häufiger als Frauen; anamnestisch läßt sich fast immer ein deutlich erhöhter Alkoholkonsum nachweisen. Innere Erkrankungen wie Leberschaden, Polyneuropathie, Diabetes mellitus, Hyperurikämie, Hyperlipidämie u.a. sind oft nachweisbar [36].

Oft wird das Lipödem mit der Adipositas verwechselt oder als Teilsymptom der Adipositas angesehen. Das Übergewicht entsteht jedoch durch eine meist weiche Fettgewebsvermehrung (epi- und supfaszial) entweder des Rumpfes (Stammfettsucht) oder des gesamten Körpers. Das Fettgewebe verursacht dabei keine Schmerzen und die Proportionen zwischen Rumpf und Extremitäten können normal sein. Männer und Frauen sind gleichermaßen betroffen. Ätiopathogenetisch sind offensichtlich genetische Faktoren (u. a. Leptin-Gen, Mutationen im Melanocortin-4-Rezeptor-Gen) sowie vor allem eine falsche bzw. eine Überernährung entscheidend [37]. Die von hypertrophierten Fettzellen sezernierten Proteine (Adipokine) werden für das Auftreten der charakteristischen Folgeerscheinungen in Form von Insulinresistenz, Dyslipidämie und Hypertonus verantwortlich 
Tab. 2 Stadien beim Lipödem

\begin{tabular}{|c|c|}
\hline Stadium I & $\begin{array}{l}\text { Subkutis verdickt und weich mit kleinen Knötchen, } \\
\text { Haut glatt }\end{array}$ \\
\hline Stadium II & $\begin{array}{l}\text { Subkutis verdickt und weich mit größeren Knoten, } \\
\text { Haut uneben }\end{array}$ \\
\hline Stadium III & $\begin{array}{l}\text { Subkutis verdickt und induriert, große Knoten, } \\
\text { wammenartige, deformierende Fettlappen an } \\
\text { Innenseiten von Oberschenkeln und Knien }\end{array}$ \\
\hline
\end{tabular}

gemacht [23]. Bei der Therapie steht die Gewichtsreduktion durch verminderte Kalorienzufuhr oder vermehrten Kalorienverbrauch (sportliche Betätigung) im Mittelpunkt. Operative Maßnahmen wie z.B. „gastric banding“ sind besonderen Fällen vorbehalten.

\section{Verlauf \\ $\nabla$}

In der Mehrzahl der Fälle nehmen die Beschwerden mit zunehmendem Alter zu; der Verlauf ist jedoch im Einzelfall nicht vorhersehbar. Eine Verschlimmerung kann einerseits durch Vermehrung des umschriebenen Unterhautfettvolumens erfolgen; diese tritt manchmal innerhalb kurzer Zeit unabhängig von bestehenden Ernährungsgewohnheiten auf. Andererseits gibt es auch Fälle, wo sich primär die Ödemneigung verstärkt. Wenn die eiweißreichen Ödeme lange Zeit unbehandelt bestehen, kann eine zunehmende Fibrosierung des Gewebes mit Ausbildung eines Lipo-Lymphödems auftreten. Wie bereits erwähnt, scheint letzteres bei adipösen Patienten schneller und öfter aufzutreten. Angaben über die Häufigkeit von Lipo-Lymphödemen bei Patienten in lymphologischen Fachkliniken reichen von 4 bis 23 Prozent [15].

\section{Therapie \\ $\nabla$}

Aufgrund der Unkenntnis des Krankheitsbildes Lipödem wurden und werden zum Teil immer noch unsinnige Therapieempfehlungen ausgesprochen. Diese beinhalten Diäten, Training der betroffenen Körperregionen oder Medikamente. Insbesondere Diäten werden aufgrund des starken Leidensdrucks von beinahe allen Patienten durchgeführt. Sie helfen allerdings nur bei Adipositas und bewirken lediglich eine Umfangsreduktion am Stamm. Da die Lipödem-spezifischen Fettvermehrungen aber nicht „abhungerungsfähig“ sind, verstärkt sich die Diskrepanz zwischen Rumpf und Extremitäten noch mehr. Auch der durch Sport erzeugte Kalorienverbrauch führt nicht zu der gewünschten Fettreduktion an den betroffenen Stellen. Sowohl der Einsatz von Abführmitteln zur möglichen Verminderung der Aufnahme von Nahrungsbestandteilen als auch von Diuretika zur Linderung ödembedingter Beschwerden ist nicht indiziert. Diuretika können allenfalls einmal kurzfristig, d.h. wenige Tage, adjuvant bei sehr starker Schwellneigung eingesetzt werden. Eine länger dauernde Gabe führt über die relative Erhöhung der Proteinkonzentration im Interstitium zu einer verstärkten Osmose und verschlimmert somit den Befund.

Die moderne Therapie des Lipödems beruht auf zwei Säulen [38]. Die konservative Behandlung hat die Beseitigung der vermehrten interstitiellen Flüssigkeitsansammlung, die operative Therapie die Reduzierung der erhöhten Unterhautfettmenge zum Ziel. Durch die Kombination beider Verfahren lassen sich
Tab. 3 a Einteilung nach Lokalisationen

$\begin{array}{ll}\text { Typ } 1 & \text { Hüften } \\ \text { Typ } 2 & \text { Hüften und Oberschenkel } \\ \text { Typ } 3 & \text { Hüften, Ober- und Unterschenkel } \\ \text { Typ } 4 & \text { Arme } \\ \text { Typ } 5 & \text { Unterschenkel }\end{array}$

Tab. 3 b Alternative Einteilung nach Lokalisationen

Oberschenkel-Typ

Unterschenkel-Typ

Waden-Typ

Ganzbein-Typ

Oberarm-Typ

Unterarm-Typ

Ganzarm-Typ

sehr gute Ergebnisse erzielen [39,40]. Da diese Behandlungen jedoch keinen Einfluss auf die gesteigerte Kapillarpermeabilität und somit auf die bestehende Ödemneigung haben, ist das Lipödem meist auf Dauer - postoperativ allerdings in deutlich geringerem Maße - therapiebedürftig.

\section{Konservative Therapie}

$\nabla$

Sie wirkt über eine Verminderung des interstitiellen Flüssigkeitsvolumens. In ganz frühen Krankheitsstadien kann bereits die Entstehung des orthostatischen Ödems durch eine Kompression in Form von Strümpfen vermieden werden. Bei nicht mehr spontan reversiblem Ödem wird die Kombinierte Physikalische Entstauungstherapie (KPE) eingesetzt.

Ihr wesentlicher Bestandteil, die manuelle Lymphdrainage (ML), bewirkt durch mit unterschiedlichem Druck durchgeführte Massagen des epifaszial gelegenen Gewebes eine Steigerung der Transportkapazität der oberflächlichen - und u. U. auch der tiefen - Lymphkollektoren. Durch Schöpf-, Dreh- und Pumpgriffe wird Ödemflüssigkeit zentripetalwärts, das heißt herzwärts verschoben. Die Behandlung wird zunächst ödemfern am Rumpf zur Erzielung eines „Sog“-Effekts und erst anschließend an den ödematösen Arealen der Extremitäten durchgeführt. Die danach angelegte Kompression mit Kurzzugbinden (Bandagierung) in Kombination mit Krankengymnastik unterstützt die Entödematisierung und verhindert die Reödematisierung.

Nach Abschluss der täglich durchgeführten Behandlung in der Ödemreduktionsphase werden bevorzugt flach gestrickte Strumpfhosen bzw. Strümpfe der Kompressionsklasse II, manchmal auch III, angepasst. In der folgenden Ödemerhaltungsphase ist die ML meist nur noch ein- bis zweimal wöchentlich notwendig. $\mathrm{Zu}$ Hause kann ergänzend die apparative intermittierende Kompression (AIK) in Form pneumatischer Mehrkammergeräte (optimal: 12 Kammern) eingesetzt werden [18,41]. Zu dieser Methode gibt es aber auch kritische Stimmen [42,43]; eine regelmäßige Kontrolle der Therapieergebnisse sollte auf jeden Fall gewährleistet sein. Für die Physikalische Ödemtherapie gelten dekompensierte Herzinsuffizienz, akute bakterielle Entzündungen und frische Thrombosen als Kontraindikationen [44].

Mit den konservativen Maßnahmen lassen sich bei den Lipödempatienten pro Bein Umfangsverminderungen von durchschnittlich 10 Prozent sowie Volumenabnahmen von bis zu 3 Litern erzielen [45]. Dies bewirkt ein Nachlassen der Spannungs- 

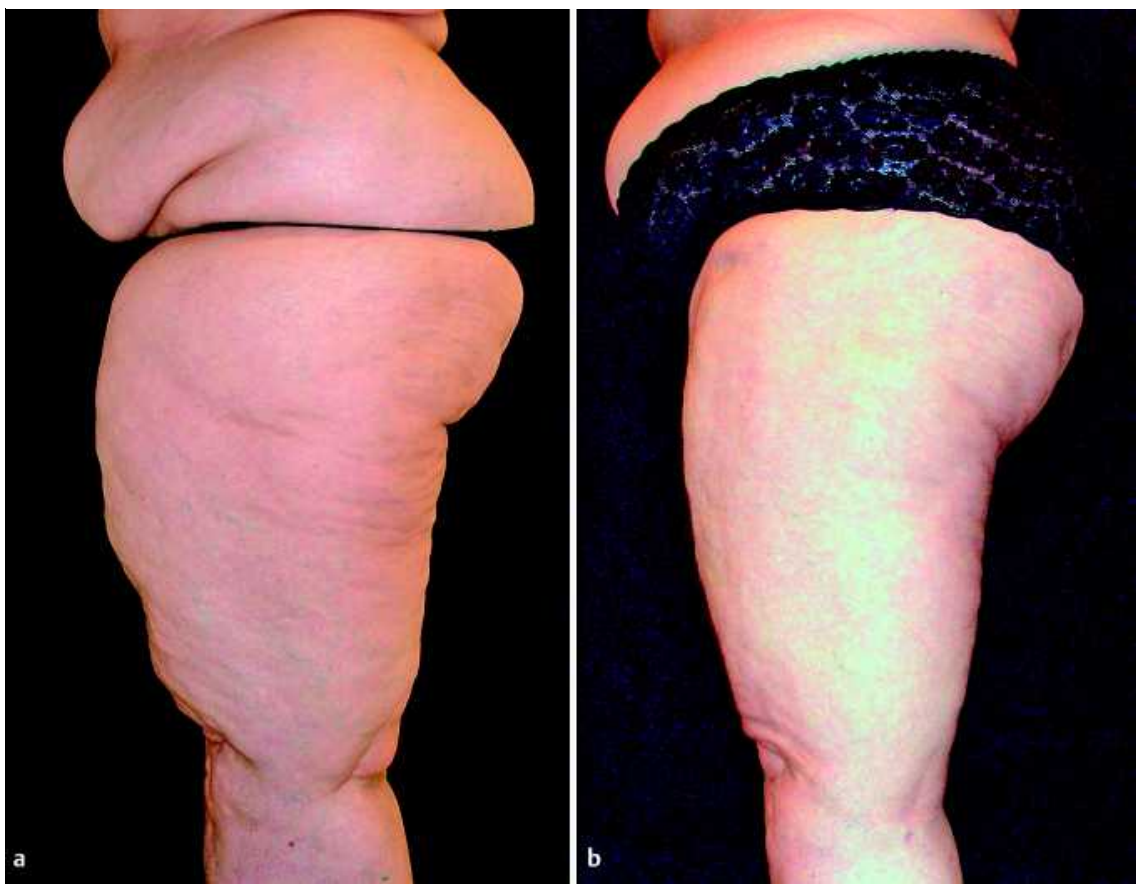

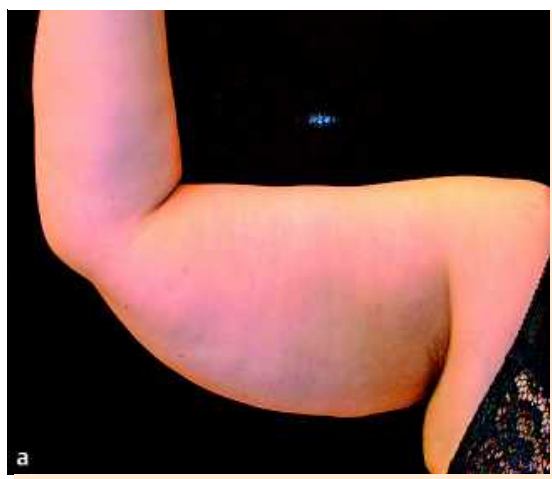

Abb. 4 Dieselbe Patientin, 38-jährig, $97 \mathrm{~kg}$. a Ausgangsbefund. b 4 Monate nach Entfernung von $1100 \mathrm{ml}$ Fett aus den Oberarmen.

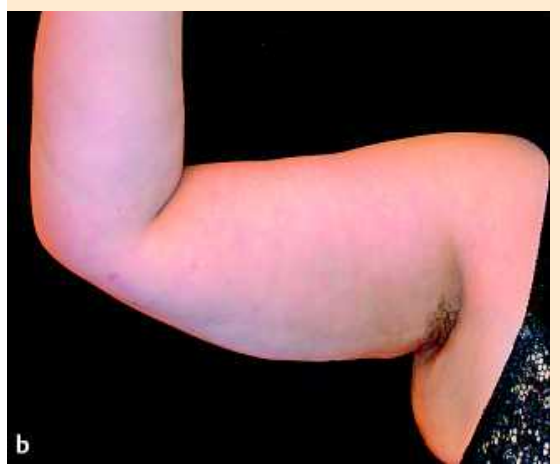

Abb. 3 Lipödem prä- und postoperativ. a Lipödem Stadium II, 37-Jährige, 109 kg, Ausgangsbefund. b Zustand 7 Monate nach Entfernung von $4150 \mathrm{ml}$ Fett aus den Oberschenkeln vorne und 1 Monat nach Entfernung von $1100 \mathrm{ml}$ Fett aus den Oberschenkeln hinten. und Druckschmerzen. Beim Aussetzen der KPE kommt es zur Nachbildung der Ödeme. Beim Lipo-Lymphödem ist das Vorgehen vom Prinzip her gleich. In ausgeprägten Fällen kann zu Behandlungsbeginn der Aufenthalt in einer lymphologischen Fachklinik sinnvoll sein.

\section{Operative Therapie}

$\nabla$

Die operative Behandlung des Lipödems hat sich erst in der letzten Zeit durchgesetzt. Bis Anfang der 1990er-Jahre wurden beim Lipödem noch großflächige Lipektomien oder Absaugungen in Vollnarkose mit dicken und vorne scharfen Absaugkanülen ohne vorherige Auffüllung des Subkutangewebes mit Flüssigkeit („dry technique“) durchgeführt. Aufgrund teilweise gefährlicher Blutungen sowie vereinzelt wohl ausgeprägter Lymphgefäßverletzungen mit persistierenden Schwellungen postoperativ wurde dieses Vorgehen von lymphologischer Seite zu Recht ablehnend beurteilt $[5,21]$.

Durch die Entwicklung neuer Betäubungs- und Operationstechniken hat in den letzten Jahren ein Wandel der Einstellung stattgefunden $[18,28,38]$. Die Risiken bzw. Nachteile einer Vollnarkose konnten durch Einführung der Tumeszenz-Lokalanästhesie (TLA) beseitigt werden [46]. Hierbei werden mehrere Liter einer 0,04\%igen Betäubungslösung mit einem Gemisch aus Lidocain und Prilocain in den Subkutanraum infiltriert („wet technique“); bei der Absaugung wird dadurch ein dünnflüssiges Fett-Lösungs-Gemisch entfernt. Vorteile dieser lokalen Betäubungsform sind die große Sicherheit für den Patienten, die Hydrodissektion (Straffung des Gewebes mit Verminderung der Scherkräfte, Lösen der Fettgewebsläppchen von den stabilisierten Bindegewebsfasern), die Hämostase (Vasokonstriktion durch den erhöhten Gewebedruck und den Adrenalinanteil in der Lösung), die langfristige postoperative Schmerzfreiheit (aufgrund der Lipophilie der Lokalanästhetika), die niedrige Infektionsrate (durch die antibakterielle Wirkung und den Auswascheffekt der postoperativ noch aus den Stichkanälen austretenden TLA-Lösung) sowie die fehlende Notwendigkeit zusätzlicher Infusionen (vorteilhaft z.B. bei kompensierter Herzinsuffizienz).

Durch den Einsatz stumpfer Mikrokanülen von 2 bis $4 \mathrm{~mm}$ Durchmesser sowie durch die Einführung der Vibrationsliposuktion lassen sich Verletzungen wichtiger Strukturen vermeiden; so konnte eine ausgeprägte Verringerung der Gewebetraumatisierung erzielt werden. Die mit hoher Frequenz $(4000 \mathrm{~Hz})$ vibrierenden Kanülen saugen nur noch das locker zwischen dem Bindegewebsgerüst liegende Fett an und schonen umliegende Strukturen wie Nerven und Gefäße weitgehend. Dies ergibt bessere kosmetische Ergebnisse sowie - aufgrund der Verminderung der Scherwirkung - eine schnellere Heilung.

Die Liposuktion hat sich inzwischen zu einem Standardverfahren entwickelt, welches - bei Durchführung unter den oben ge- 

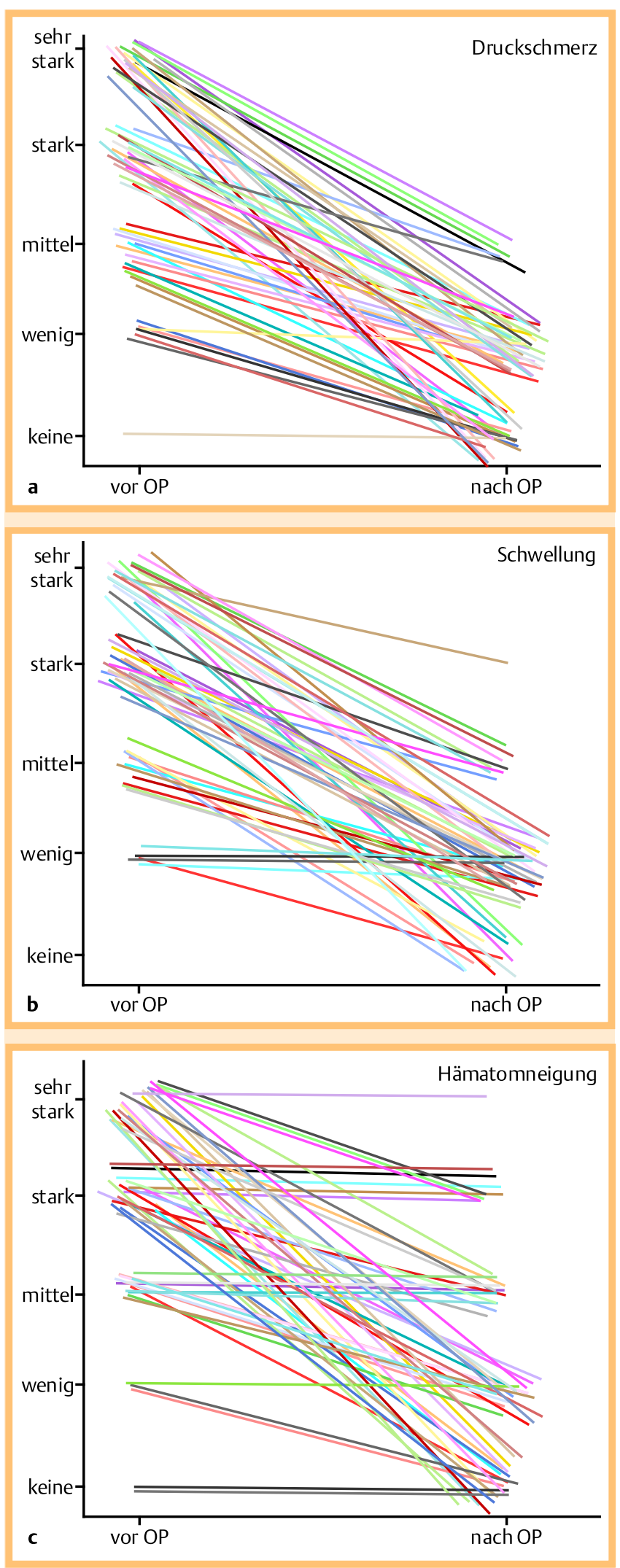

schilderten Bedingungen - sehr wirksam und ausgesprochen sicher ist [47]. Die in der Literatur geschilderten Komplikationen und z.T. auch Todesfälle waren überwiegend Folge der fehlenden Beachtung international etablierter Richtlinien und manchmal auch fehlender medizinischer Basiskenntnisse des Operateurs $[48,49]$. Da pro Eingriff möglichst nicht mehr als 4 Liter reines

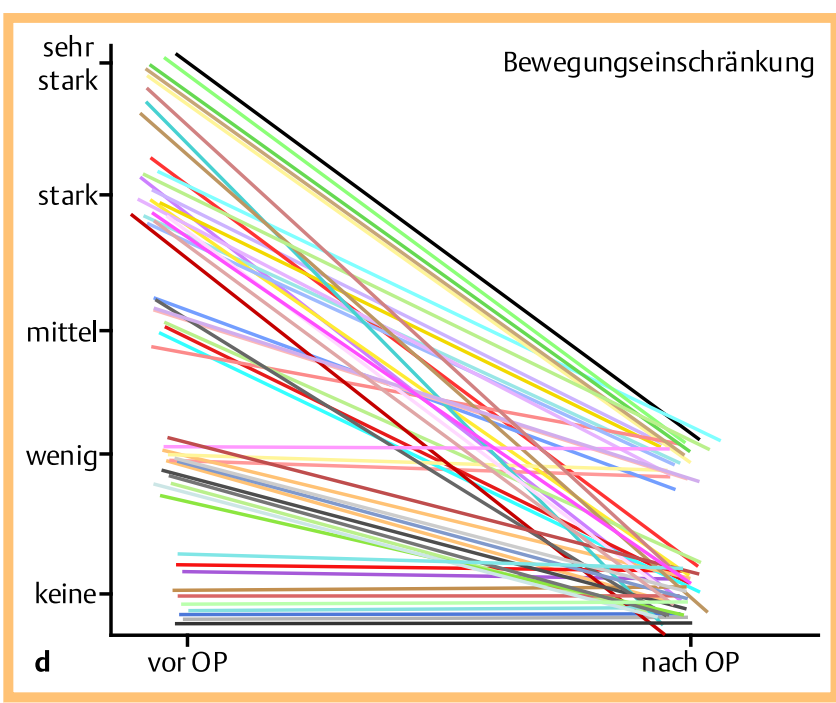

Abb. 5 Selbsteinschätzung der Befundbesserung bei 71 Patientinnen präoperativ und durchschnittlich 13,2 (Spannbreite 1 -55) Monate postoperativ. a Druckschmerz. b Schwellung. c Hämatomneigung. d Bewegungseinschränkung.

Fett entfernt werden sollte, sind je nach Ausmaß des Befundes meist zwischen ein und vier Operationen im Abstand von mehreren Monaten notwendig ( $\bullet$ Abb. 3 u. 4). Damit kann man einerseits das äußere Erscheinungsbild der Patientinnen optimieren und wieder harmonische Körperproportionen herstellen; andererseits lassen sich - über die Reduzierung der epifaszial gelegenen Fettvolumina - auch die Ödeme und damit die Schmerzempfindlichkeit des Gewebes beseitigen oder zumindest deutlich reduzieren $[16,40,50]$. Da postoperativ meist kurzfristig eine vermehrte Schwellneigung auftritt, sollte die physikalische Therapie bereits kurz nach dem Eingriff begonnen bzw. weitergeführt werden.

Die früher teilweise beschriebenen Lymphgefäßschädigungen durch die Liposuktion mit nachfolgendem Auftreten von Lymphödemen ließen sich bei Einsatz der neuen Methoden bisher weder experimentell noch klinisch beobachten. So konnten anatomische Untersuchungen nach Absaugung in Längsrichtung der Extremitäten makroskopisch keine Schädigungen epifaszialer Lymphgefäße nachweisen [51,52]. Bei immunhistologischen Untersuchungen im abgesaugten Aspirat zeigten sich zwar Wandfragmente von Blutkapillaren, nicht jedoch von Lymphgefäßen [53].

In der Literatur berichtete klinische Nachbeobachtungen mit bisher noch geringen Zahlen (19 Patientinnen) über einen Zeitraum von 8 Jahren zeigten weder eine vermehrte Schwellneigung noch eine Progredienz der Erkrankung [50]. Bezüglich der eigenen Ergebnisse kann gesagt werden, dass derzeit die operative Behandlung bei 71 Lipödempatientinnen abgeschlossen ist; insgesamt wurden dabei 175 Eingriffe (durchschnittlich 2,46 (Spannweite 1-7) pro Patient) durchgeführt. Bei diesem Kollektiv kann ein Nachbeobachtungszeitraum von bis zu 4 1/2 Jahren (durchschnittlich: 13,2 Monate) überblickt werden.

Bezüglich des postoperativen Verlaufs gaben 25 Prozent der Patientinnen an, keine weitere Kompression und auch keine Lymphdrainage mehr zu benötigen; 41 Prozent erklärten, dass die Weiterführung der konservativen Therapie in deutlich verringertem Ausmaß nötig war. 23 Prozent beschrieben - bei weitgehender Beibehaltung von Kompression und Lymphdrainagen - eine deutliche Besserung der Lebensqualität; die konservative 
Behandlung wurde postoperativ als wesentlich wirksamer beschrieben. 11 Prozent hatten erst nach der Diagnosestellung bei uns von der Möglichkeit einer Lymphdrainage erfahren bzw. deren Vorteile kennen gelernt. Die $\bullet$ Abb. 5 a -d zeigen die Selbsteinschätzungen der operierten Patienten.

Leider werden die Kosten für die Liposuktion bisher überwiegend nicht von den gesetzlichen Krankenkassen übernommen.

\section{Abschließende Bemerkung \\ $\nabla$}

Die Behandlung des Lipödems hat in den letzten 10 Jahren einen entscheidenden Wandel erfahren. Durch die Kombination operativer und konservativer Maßnahmen können heute optimale Ergebnisse erzielt werden. Die Fettabsaugung sollte frühzeitig durchgeführt werden, um durch Verminderung der Zahl der betroffenen Fettzellen die Progredienz des Krankheitsbildes zu bremsen. Damit allein ist die Erkrankung jedoch meist nicht vollständig zu beseitigen. Entscheidend ist das Ausmaß der Ödemneigung.

Die Liposuktion kann zwar die Fettmenge vermindern bzw. normalisieren; sie hat jedoch keinen Einfluss auf die bestehende Kapillardurchlässigkeit. Die von den Betroffenen bemerkte Verminderung der Schwellneigung postoperativ ist wahrscheinlich Folge der Verkleinerung des epifaszialen Raums. Nach der Absaugung kann - unabhängig vom Stadium der Erkrankung - bei etwa einem Viertel der Fälle eine vollständige Beschwerdefreiheit erzielt werden; bei den übrigen Patientinnen existiert aber immer noch eine gewisse Ödemneigung. Daher muss die Basistherapie in Form der KPE - allerdings überwiegend in deutlich verringerter Frequenz und mit geringerer Kompressionsstärke weitergeführt werden.

Entscheidend für den Dermatologen ist somit - nach richtiger Diagnosestellung - die Weiterleitung der Patientinnen an ausgebildete Lymphtherapeuten sowie an Operateure, die mit der Liposuktion bei diesem Krankheitsbild vertraut sind.

\section{Abstract}

\section{Lipoedema - New Facts of a Fairly Unknown Disease $\nabla$}

Lipoedema, a disease in women, is characterized by circumscribed accumulation of subcutaneous fat, mainly in the legs, with oedema, pain by tension and pressure and hematomas. Diagnosis can be achieved by clinical signs and symptoms. Up to now, only conservative therapy by manual lymphatic drainage, compression and physiotherapy was possible. Nowadays, surgical therapy with liposuction in tumescent local anaesthesia (TLA) can improve quality of life considerably by correcting morphology and discomfort. The combination of both therapeutic regimens is the new standard of treatment.

\section{Literatur}

1 Allen EU, Hines EA. Lipedema of the legs: A syndrom characterized by fat legs and orthostatic edema. Proc Staff Mayo Clin 1940; 15: 184187

2 Rudkin GH, Miller TA. Lipedema: a clinical entity distinct from lymphedema. Plast Reconstr Surg 1994; 94: 841 - 847

3 Witte MH, Witte CL. Massive obesity simulating lymphedema. N Engl J Med 1992; 327: 1927
4 Koss T, Lanatra N, Stiller MJ, Grossmann ME. An unusual combination: Lipedema with Myiasis. J Am Acad Dermatol 2004; 50: 969-972

5 Strößenreuther RHK (Hrsg.). Lipödem und andere Erkrankungen des Fettgewebes. Köln: Viavital, 2001

6 Fritsch P. Dermatologie und Venerologie. Grundlagen, Klinik, Atlas, 2. Aufl. Berlin, Heidelberg: Springer, 2003

7 Meves A. Intensivkurs Dermatologie. München: Elsevier, 2006

8 Rassner G, Steinert U. Dermatologie, Lehrbuch und Atlas. 8. Aufl. München: Elsevier, 2007

9 Altmeyer P, Bacharach-Buhles M. Dermatologie, Allergologie, Umweltmedizin. Berlin: Springer, 2002

10 Moll I. Duale Reihe. Dermatologie, 6. Aufl. Stuttgart: Thieme, 2005

11 Sterry W, Paus R. Checkliste Dermatologie, Venerologie, Phlebologie, Andrologie, 5. Aufl. Stuttgart: Thieme, 2004

12 Netter FH. Netters Dermatologie. Stuttgart: Thieme, 2006

13 Braun-Falco O, Plewig G, Wolff HH, Burgdorff WHC, Landthaler M (Hrsg). Dermatologie und Venerologie, 5. Aufl. Berlin, Heidelberg, New York: Springer, 2005: 1022 - 1023

14 Rabe E, Pannier-Fischer F. Venenerkrankungen. In: Hengge U, Ruzicka T (Hrsg). Lehrbuch der Dermatologie und Venerologie. Stuttgart: Wissenschaftliche Verlagsgesellschaft, 2006: 338-347

15 Meier-Vollrath I, Schneider W, Schmeller W. Lipödem. Verbesserte Lebensqualität durch Therapiekombination. Dt Ärzteblatt 2005; 102 : A-1061 - A-1067, B-892 - B-897, C-840-C-845

16 Chen S, Hsu SD, Chen TM, Wang HJ. Painful fat syndrome in a male patient. Br J Plast Surg 2004; 57: 282 - 286

17 Wold LE, Hines EA, Allen EV. Lipedema of the legs: A syndrom characterized by fat legs and edema. Ann Intern Med 1949; 34: 1243-1250

18 Marshall M, Breu FX. Das Lipödem - ein wenig beachtetes Krankheitsbild. Vasomed 2002; 6: 254-257

19 Herpertz U. Entstehungszeitpunkt von Lipödemen. LymphForsch 2004; 8: 79-81

20 Harwood CA, Bull RH, Evans J, Mortimer PS. Lymphatic and venous function in lipedema. Br J Dermatol 1996; 134: 1-6

21 Földi E, Földi M. Das Lipödem. In: Földi M, Kubik S (Hrsg). Lehrbuch der Lymphologie für Mediziner, Masseure und Physiotherapeuten, 6. Aufl. München, Jena: Urban \& Fischer, 2005: 444- 453

22 Kaiserling E. Morphologische Alterationen. In: Földi M, Kubik S (Hrsg). Lehrbuch der Lymphologie für Mediziner, Masseure und Physiotherapeuten, 6. Aufl. München, Jena: Urban \& Fischer, 2005: 331 - 388

23 Fasshauer M, Klein J, Blüher M, Paschke R. Adipokine: Mögliches Bindeglied zwischen Insulinresistenz und Adipositas. Dtsch Ärzteblatt 2004; 101: A3491 - A3495

24 Greer KE. Lipedema of the legs. Cutis 1974; 14: 98 - 100

25 Weissleder H, Brauer WJ. Radiologische Diagnostik beim LipödemSyndrom. LymphForsch 1997; 1: 26-30

26 Bilancini S, Lucchi M, Tucci S, Eleuteri P. Functional lymphatic alterations in patients suffering from lipedema. Angiology 2002; 46: $333-$ 339

27 Brauer WJ, Weissleder H. Methodik und Ergebnisse der Funktionslymphszintigraphie: Erfahrungen bei 924 Patienten. Phlebologie 2002; 31: $118-125$

28 Herpertz $U$. Ödeme und Lymphdrainage. Diagnose und Therapie von Ödemkrankheiten, 3. Aufl. Stuttgart, New York: Schattauer, 2006: $168-181$

29 Herpertz $U$. Krankheitsspektrum des Lipödems an einer Lymphologischen Fachklinik - Erscheinungsformen, Mischbilder und Behandlungsmöglichkeiten. Vasomed 1997; 5: 301 - 307

30 Dimakakos PB, Stefanopoulos T, Antoniades P, Antoniou A, Gouliamos A, Rizos $D$. MRI and ultrasonographic findings in the investigation of lymphoedema and lipedema. Int Surg 1997; 82: 411 - 416

31 Monnin-Delhom ED, Gallix BP, Achard C, Bruel JM, Janbon C. High resolution unenhanced computed tomography in patients with swollen legs. Lymphology 2002; 35: $121-128$

32 Breu FX, Marshall M. Neue Ergebnisse der duplexsonographischen Diagnostik des Lip- und Lympödems. Kompressionssonographie mit einer neuen 13-MHz-Linearsonde. Phlebologie 2000; 29: 124-128

33 Amman-Vesti BR, Fanzeck UK, Bollinger A. Microlymphatic aneurysms in patients with lipedema. Lymphology 2001; 34: 170-175

34 van Geest AJ, Esten SCAM, Cambier JPRA, Gielen EGJ, Kessels A, Neuman $H A M$, van Kroonenburgh MJPG. Lymphatic disturbances in lipoedema. Phlebologie 2003; 32: 138 - 142

35 Steiner J, Schiltz K, Heidenreich F, Weissenborn K. Lipomatosis dolorosa - ein häufig übersehenes Krankheitsbild. Nervenarzt 2002; 73: 183 187 
36 Sehouli J, Stengel D, Schauwecker H. Benigne symmetrische Lipomatose. Therapeutisches Dilemma? Langenbecks Arch Chir 1997; 382: $271-273$

37 Hebebrand J, Dabrock P, Lingenfelder M, Mand E, Rief W, Voit W. Ist Adipositas eine Krankheit? Dtsch Ärzteblatt 2004; 101: A 2468 -A 2474

38 Wienert V, Földi E, Schmeller W, Rabe E. Leitlinie: Lipödem der Beine. Phlebologie 2005; 34: $38-40$

39 Schmeller W, Meier-Vollrath I. Anmerkungen zur Therapie des Lipödems. LymphForsch 2006; 10: $22-26$

40 Schmeller W, Meier-Vollrath I. Das Lipödem: neue Möglichkeiten der Therapie. SchweizMedForum 2007; 7: 150-155

41 Rabe E (Hrsg). Apparative intermittierende Kompressionstherapie (AIK). Köln: Viavital, 2003

42 Gültig 0 . Erfolg und Mißerfolg bei Einsatz der Apparativen Intermittierenden Kompressionstherapie (AIK) - Ergebnisse einer breit angelegten Fragebogenaktion bei Patienten mit chronischem Lymphödem der Extremitäten. LymphForsch 2004; 8: 91 - 92

43 Weissleder H. AIK und Lymphödem - Glaube und Wirklichkeit. LymphForsch 2004; 8: 93-95

44 Schneider W, Herpertz U. Indikation und Kontraindikation der physikalischen Ödemtherapie. Orthopädie-Technik 1996; 3: 185-191

45 Deri G, Weissleder $H$. Vergleichende prä- und posttherapeutische Volumenmessungen in Beinsegmenten beim Lipödem. Lymph Forsch 1997: $1: 35-37$
46 Klein JA. The tumescent technique. Anesthesia and modified liposuction technique. Dermatol Clin 1990; 8: 425-437

47 Sattler G, Sommer B, Hanke CW. Leitlinien zur Liposuktion. In: Sattler G, Sommer B, Hanke CW (Hrsg). Lehrbuch der Liposuktion. Stuttgart: Thieme, 2003: 217 - 222

48 Lehnhardt M, Homann HH, Druecke D, Palka P, Steinau HU. Liposuktion - kein Problem? Majorkomplikationen und Todesfälle im deutschen Raum zwischen 1998 und 2002. LymphForsch 2004; 8: 74 - 78

49 Schmeller W, Meier-Vollrath I. Kommentar zum Artikel: Komplikationen nach Liposuktion von Berroth R, Speichermann N, Liebau G. Intensivmed 2004; 41: 64-66

50 Rapprich S, Loehnert M, Hagedorn M. Therapy of lipoedema syndrome by liposuction under tumescent local anaesthesia. Ann Dermatol Venereol 2002; 129: 1 S711

51 Frick A, Hoffmann JN, Baumeister RGH, Putz R. Liposuction technique and lymphatic lesions in lower legs: Anatomic study to reduce risks. Plast Reconstr Surg 1999; 103: 1868-1873

52 Hoffmann JN, Fertmann JP, Baumeister RG, Putz R, Frick A. Tumescent and dry liposuction of lower extremities: differences in lymph vessel injury. Plast Reconstr Surg 2004; 113: 718 - 724; discussion 725-726

53 Schmeller W, Tronnier M, Kaiserling E. Lymphgefäßschädigung durch Liposuktion? Eine immunhistologische Untersuchung. LymphForsch 2006; 9: $81-85$ 